\title{
Autophagy and Apoptosis Associated with Abortive Pollen Development in the Cytoplasmic Male-sterile Line GP1BC1-12 of Platycodon grandiflorum
}

\author{
Fenghua Shi \\ Institute of Medicinal Plant Development (IMPLAD), Chinese Academy of Medical Sciences \& Peking \\ Union Medical College, No. 151, Malianwa North Road, Haidian District, Beijing 100193, China; \\ and Guangxi Botanical Garden of Medicinal Plants, Nanning 530023, China
}

\author{
Chun Sui and Yue Jin \\ Institute of Medicinal Plant Development (IMPLAD), Chinese Academy of Medical Sciences \& Peking \\ Union Medical College, No. 151, Malianwa North Road, Haidian District, Beijing 100193, China
}

\author{
Hao Huang \\ Guangxi Botanical Garden of Medicinal Plants, Nanning 530023, China
}

\begin{abstract}
Jianhe Wei ${ }^{1}$
Institute of Medicinal Plant Development (IMPLAD), Chinese Academy of Medical Sciences \& Peking Union Medical College, No. 151, Malianwa North Road, Haidian District, Beijing 100193, China
\end{abstract}

\begin{abstract}
AdDitional INDEX words. abortive anthers, balloon flower, medicinal plant, programmed cell death, terminal deoxynucleotidyl transferase-mediated deoxyuridine triphosphate nick end labeling assay

Abstract. Platycodon grandiflorum (balloon flower) is widely cultivated for medicinal, edible, and ornamental purposes. The cytoplasmic male-sterile line GP1BC1-12 of $P$. grandiflorum has been used to produce hybrids, but its mechanism of sterility has not been studied. In this work, the mechanism was evaluated by a combination of transmission electron microscopy (TEM) and terminal deoxynucleotidyl transferase-mediated deoxyuridine triphosphate nick end labeling assay. Observations showed that autophagy and apoptosis were simultaneously present in the abortive anthers. Autophagy was indicated by the autophagic vesicles, abnormal arrangement of endoplasmic reticulum, and the vacuole's invagination. Apoptosis was characterized by chromatin aggregation and DNA cleavage. It was concluded that programmed cell death is one of the direct reasons for cytoplasmic male sterility in $P$. grandiflorum. This study first noted the simultaneous presence of the features of apoptosis, microautophagy, and macroautophagy in the abortive anthers of $P$. grandiflorum.
\end{abstract}

Platycodon grandiflorum is widely grown as a garden plant and is a source of cut flowers in Europe and the United States (Halevy et al., 2002; Park et al., 1998; Song et al., 1993). It is cultivated in southeast Asia as vegetables and herbs. Its roots are a traditional Chinese medicine for the treatment of cough with much phlegm, sore throat, hoarseness, and pulmonary abscesses with pus-running abscesses that only burst with difficulty after suppuration (Liu and Ma, 2001). P. grandiflorum, as a popular vegetable, has received much attention to be a functional food (Choi et al., 2010; Kang et al., 2008). Genetic deterioration and severe diseases have resulted from the longterm use of native germplasm in most $P$. grandiflorum-growing districts of China. New cultivars are urgently needed to produce $P$. grandiflorum roots with high yield and good quality.

As an effective approach, heterosis has been widely used to produce cultivars for crops, vegetables, and grasses (Patel et al., 2009; Pradhan et al., 1993; Vogel and Mitchell, 2008). The study

Received for publication 24 Feb. 2014. Accepted for publication 15 Apr. 2014. This work was financed by the National Natural Science Foundation of China (Hrant No. 31260353), the Guangxi Natural Science Foundation of China (No. 2012GXNSFDA053019), and Program for Innovative Research Team in IMPLAD.

We thank Dr. Edward C. Mignot from Shandong University for linguistic advice.

${ }^{1}$ Corresponding author. E-mail: wjianh@263.net. of heterosis use of the male-sterility system in $P$. grandiflorum was started in our laboratory in 2004. Some cytological malesterile lines have been developed from naturally male-sterile plants by backcrosses. Among them, the cytoplasmic male-sterile (CMS) line GP1BC1-12 has the highest sterility rate and the best performance in the field. Three hybrids of $P$. grandiflorum with high yield and high disease resistance, Zhonggeng No. 1, Zhonggeng No. 2, and Zhonggeng No. 3, were derived from it (Shi et al., 2011; Wu et al., 2008, 2009b). To provide necessary information for further use of the CMS line GP1BC1-12, its mechanism of male sterility has to be understood.

The mechanism of male sterility has been widely studied. An anatomical abnormality in the tapetum and its organelles has been implicated directly or indirectly leading to abortion of microsporocyte, tetrad, microspore, or pollen grain. Recently, the study focus of male sterility has shifted to the programmed cell death (PCD) of pollen development (Coimbra et al., 2004; $\mathrm{Ku}$ et al., 2003; Shi et al., 2009; Wan et al., 2010). PCD is an active cell death process involved in the selective elimination of unwanted cells (Greenberg, 1996, 1997; Vaux and Korsmeyer, 1999). It is essential throughout a plant's life cycle, especially in the reproductive phase (Henming and Alice, 2000; Love et al., 2008; Pennell and Lamb, 1997; Wu and Cheung, 2000). Such are all the processes of PCD, for example, crushing of the middle layer in early meiosis, the disintegration of the tapetum 
soon after pollen development for releasing their lipid components, and the degradation of the intersporangial septum and stomium cells of the anthers to allow anther dehiscence (Greenberg, 1996; Henming and Alice, 2000). The PCD has been found during the development of anthers in male-sterile plants such as Helianthus annuus, Oryza sativa, Arabidopsis thaliana, and Castanea sativa (Balk and Leaver, 2001; Li et al., 2006; Vizcay-Barrena and Wilson, 2006; Zhang et al., 2007).

Apoptosis, autophagy, and necrosis are morphological types of cell death. PCD is usually used to describe the processes of apoptosis and autophagy (Hayward et al., 2009; Reape et al., 2008; Van-Doorn and Woltering, 2005). Chromatin condensation and DNA fragmentation are the two characteristics of apoptosis in both animals and plants (Krishnamurthy et al., 2000; Van-Doorn et al., 2011; Wyllie, 1980). DNA fragmentation has been successfully detected showing premature or delayed PCD in the tapetal cells of sterile anthers and has been taken as the hallmark of apoptosis. Apoptosis of tapetal cells at an abnormal stage is considered to be the main cause of male sterility (Balk and Leaver, 2001; Ku et al., 2003).

Autophagy is the other course for non-apoptotic death of PCD, by which portions of the cytoplasm are sequestered and further degraded by lytic vacuolar enzymes (Edinger and Thompson, 2004). Two types of autophagic processes have been described: macroautography and microautography. The difference between them is the way by which the cytoplasm is sequestered. The former is characterized by formation of the vacuole in which portions of the cytoplasm are sequestered. The latter is manifested by invagination of the tonoplast and the subsequent engulfment of cytoplasmic fragments into the cavity of a pre-existing vacuole (Aubert et al., 1996). Autophagy is shown to be involved in developmental processes such as vacuole formation, deposition of seed storage proteins and senescence, and plant response to nutrient starvation and pathogens (Bassham, 2007; Bassham et al., 2006). However, it was seldom found in male-sterile materials, except that it was evaluated by indirect evidence of membrane-bound inclusions in male sterility of Brassica napus (Wu et al., 2009a) without direct evidence of the autophagic vesicle or the invagination of the tonoplast.

To detect if PCD is involved with male sterility in the CMS line GP1BC1-12, an ultrastructural comparison and terminal deoxynucleotidyl transferase-mediated deoxyuridine triphosphate nick end labeling (TUNEL) experiment was conducted for the fertile and the sterile lines during pollen development. This study first noted that apoptosis and autophagy were simultaneously present in the microsporocyte and the tapetal cells of sterile anthers at the microsporocyte stage and meiosis stage. The microautophagy and macroautophagy in sterile anthers support the conclusion that PCD in plants has the typical morphological features of autophagy (Bassham, 2007; Bassham et al., 2006).

\section{Materials and Methods}

Plants and growth conditions. Seeds of maintainer line GS902 (fertile plant) and CMS line GP1BC1-12 (sterile plant) were sown with a $25-\mathrm{cm}$ row width and $50-\mathrm{cm}$ plant spacing in the paddy field of the Institute of Medicinal Plant Development in Beijing. A total of 144 plants for each of GS902 and GP1BC1-12 were finally obtained. Buds in different length from 2 to $13 \mathrm{~mm}$ were randomly collected from the 144 GS902 individual plants and $144 \mathrm{GP} 1 \mathrm{BC} 1-12$ individual plants, respectively. One anther was picked from each bud and was checked for microspore development stage under light microscopy. Finally, for both GS902 and GP1BC1-12 lines, 10 anthers were stored for further use in microsporocyte stage, meiosis stage, and metaphase, respectively.

UltrastruCTURE OBSERVATION OF ANTHERS. For both GS902 and GP1BC1-12 lines, five anthers were selected in microsporocyte stage, meiosis stage, and metaphase, respectively. Each anther was made as an embedding block. Three blocks with anthers in microsporocyte stage, meiosis stage, and metaphase, respectively, were randomly selected and cut into slices for observation. Anthers were fixed in 3\% (w/v) paraformaldehyde and $0.25 \%$ glutaraldehyde in $0.2 \mathrm{~N}$ sodium phosphate buffer, $\mathrm{pH}$ 7.0 , and then in $2 \% \mathrm{OsO}_{4}$ in phosphate-buffered solution (PBS), $\mathrm{pH}$ 7.2. After ethanol dehydration, samples were embedded in acrylic resin. Ultrathin sections $(70 \mathrm{~nm})$ were double-stained with $2 \%(\mathrm{w} / \mathrm{v})$ uranyl acetate and $2.6 \%(\mathrm{w} / \mathrm{v})$ lead citrate aqueous solutions and examined with a TEM (JEM-1400; JEOL, Tokyo, Japan) at $80 \mathrm{kV}$.

TUNEL ASSAY. For both GS902 and GP1BC1-12 lines, five anthers were embedded and cut into slices as for ultrastructure observation of anthers in microsporocyte stage, meiosis stage, and metaphase, respectively. Anthers were dissected from flowers and fixed overnight in FAA solution (50\% ethanol, 5\% glacial acetic acid, 5\% formaldehyde), dehydrated in a graded ethanol series, cleared of chlorophyll II, embedded in paraffin, and sliced into $8-\mu \mathrm{m}$ sections. After dewaxing and rehydration, paraffin sections of anthers were rinsed in PBS washing solution (160 mm NaCl, $2.7 \mathrm{~mm} \mathrm{KCl}, 8 \mathrm{~mm} \mathrm{Na}_{2} \mathrm{PO}_{4}$, and $1.5 \mathrm{~mm} \mathrm{KH}_{2} \mathrm{PO}_{4}$ ) for $5 \mathrm{~min}$ and incubated in $20 \mu \mathrm{g} \cdot \mathrm{mL}^{-1}$ proteinase $\mathrm{K}$ in $10 \mathrm{~mm}$ Tris-HCl, pH 7.4 to 8 , for $30 \mathrm{~min}$. The PBS washing was repeated. In situ nick-end labeling of nuclear DNA fragments was performed in a humid chamber for $1 \mathrm{~h}$ in the dark at $37^{\circ} \mathrm{C}$ with a TUNEL apoptosis detection kit (In Situ Cell Death Detection Kit; Roche, Mannheim, Germany) according to the supplier's instructions. After being washed in PBS washing solution three times, $50 \mu \mathrm{L}$ converter-peroxidase was added to the samples and then the slides were incubated in a humidified chamber for $30 \mathrm{~min}$ at $37^{\circ} \mathrm{C}$. The slides were again washed with PBS washing solution, and 50 to $100 \mu \mathrm{L}$ diaminobenzidine substrate was added to the samples. The samples were then counterstained by $0.5 \%$ fast green and analyzed under a light microscope.

\section{Results}

The plants of CMS line GP1BC1-12 were normal in floral development but failed to produce any viable pollen. Its stamens were only half as long as its stigma, and no anther sacs were found in its mature anthers. To gain a more detailed understanding of the abnormalities of the CMS line GP1BC1-12, TEM observation and TUNEL assay were used to study the sterile anther with fertile anther as control.

Ultrastructural features of PCD in microsporocytes AND TAPETAL CELlS IN THE MiCrosporocyte STAGE. In the microsporocyte stage, for the fertile anther, the microsporocyte was occupied by an approximately round nucleus with a nucleolus in its middle. Its cytoplasm had mitochondria, plastids, and starch granules (Fig. 1A). The endoplasmic reticulum (ER) was discrete, short, and straight (Fig. 1B). Tapetal cells at this stage had a single large vacuole that occupied most of the cellular 


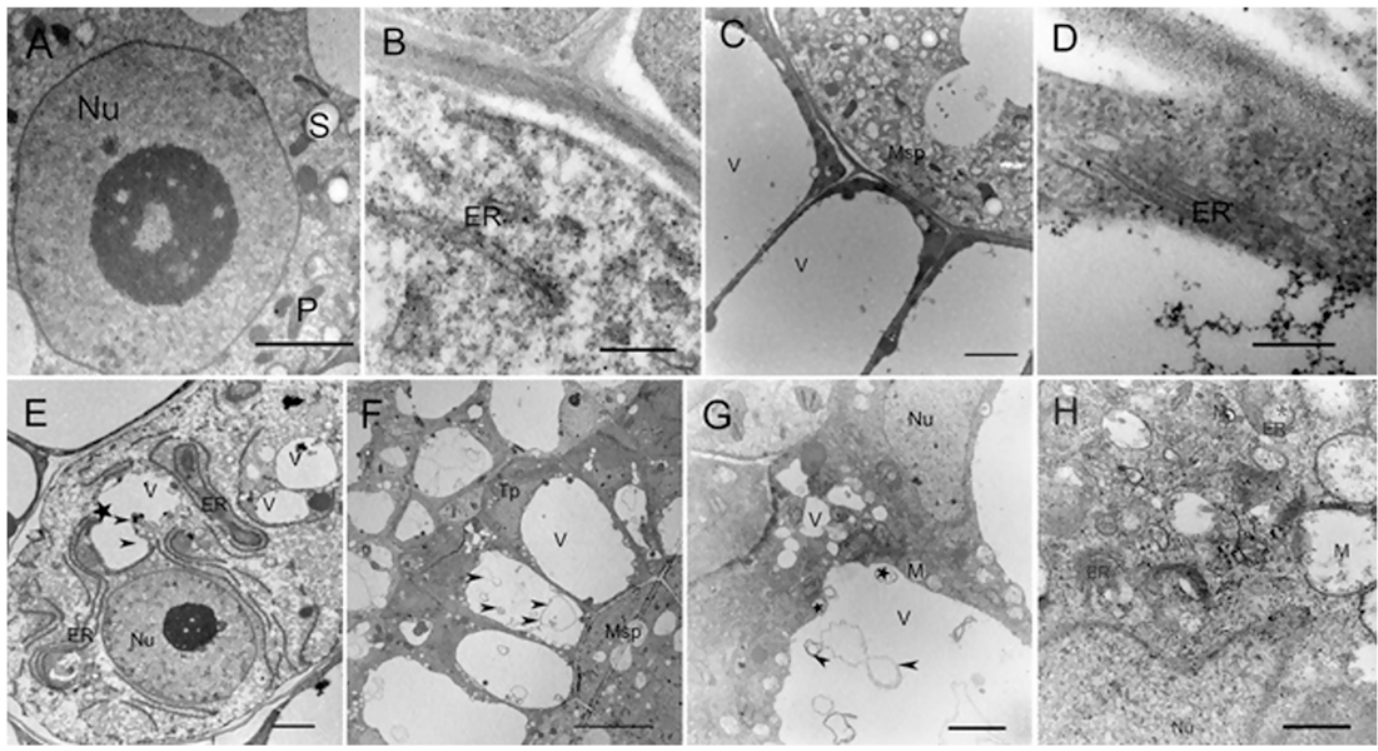

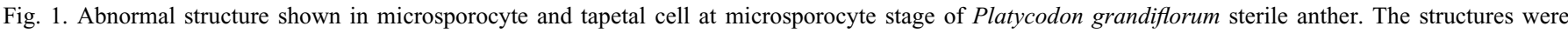
observed by transmission electron microscope at microsporocyte stage of anthers from cytoplasmic male sterile line GP1BC1-12 and maintainer fertile line GS902. Comparing the normal nucleus (A) and endoplasmic reticulum (ER) (B) of the microsporocyte in fertile anther, aggregative chromatin and elongated ER with some organelles encompassed in it were present in the microsporocyte of sterile anther (E). The ruptured tonoplast (asterisk) and an invagination (arrows) in vacuole were detected in the abnormal microsporocyte (E). In tapetal cell of fertile anther, the tonoplast of the tapetal cell was slide (C) but blebbed in sterile anther. Some lamellar (arrows) was present in vacuole (F). Two mitochondria (asterisks) were detected to be absorbed into vacuole (G). ER of the tapetal cell in fertile anther was present with a pattern of short stalks (D), but concentric pattern (asterisk) in sterile anther $(\mathbf{H})$ and swollen mitochondria were also detected (H). The scale bars are $2 \mu \mathrm{m}$ for $\mathbf{A}, \mathbf{C}, \mathbf{E}$, and $\mathbf{G}, 0.5 \mu \mathrm{m}$ for $\mathbf{B}, 0.2 \mu \mathrm{m}$ for $\mathbf{D}, 1 \mu \mathrm{m}$ for $\mathbf{H}$, and $10 \mu \mathrm{m}$ for $\mathbf{F} ; \mathbf{M}=\mathrm{mitochondria} ; \mathrm{Msp}=\mathrm{microsporocyte}$; Nu= nucleus; $\mathbf{P}=$ plastid; $\mathrm{S}=$ starch granule; $\mathrm{Tp}=$ tapetum; $\mathrm{V}=$ vacuole.

volume (Fig. 1C), and the ER had short stalks (Fig. 1D). In the sterile anther, however, abnormal structures were present everywhere in both the microsporocyte and tapetal cells. Its microsporocyte also had a round nucleus with a nucleolus as in a normal microsporocyte, but heterochromatin resulted from chromatin aggregation scattered around the edge of the nucleus. An invagination has formed in the surface of a vacuole with part of the cytoplasm seemingly sequestrated by this vacuole. The ER was in a different arrangement of inclusion and elongation, and part of the cytoplasm and some organelles such as a vacuole were included in it (Fig. 1E). The tapetal cells of the sterile anther also had a single vacuole as in normal cells; however, some lamellae were present in it (Fig. 1F). A mitochondria was being autophaged and another had been completely eaten by the vacuole (Fig. 1G). Its mitochondria in the tapetal cell were swollen with the damaged inner membrane, and the ER was in a concentric pattern in appearance that is different from the straight pattern of normal ER in the tapetal cell of fertile anther. Furthermore, a mitochondrion was seemingly surrounded by these stacks of ER (Fig. 1H).

ULTRASTRUCTRAL FEATURES OF PCD IN MICROSPOROCYTE AND TAPETAL CELLS IN THE MEIOSIS STAGES AND THE METAPHASE. At the onset of meiosis, for the fertile anther, each microsporocyte secretes a callose wall around itself, and the cytoplasm with some small vacuoles had a dense electron staining (Fig. 2A). The tapetal cell at this stage had a dense cytoplasm and big vacuoles (Fig. 2B), mitochondria and plastid in its cytoplasm looked healthy, and the ER consisted of a few short stalks (Fig. 2C). For the sterile anther, however, the whole microsporocyte layer was hollow except the remaining vestigium (Fig. 2D). In the vestigium of the microsporocyte, no cellular structure was discerned (Fig. 2E). Some tapetal cells have comparatively integral structure, and another tapetal cells had scarcely any organelles (Fig. 2D). In the tapetal cells with a comparatively integral structure, their mitochondria and plastids looked healthy, and their nuclear membrane was intact, but chromatin aggregation occurred in their nucleus (Fig. 2F). Two abnormal structures were usually present in these tapetal cells: the ER with an elongated pattern in which part of cytoplasm was surrounded and vesicles arose from the edge of the vacuole (Fig. 2G). The ER with an elongated pattern; these vesicles also contained an ER-like substance in it (Fig. 2H). Interestingly, multilamellar vesicles, detected in the Acer pseudoplatanus cell which were deprived of sucrose (Aubert et al., 1996), was also observed during the degradation of tapetal cell of P. grandiflorum (Fig. 2I). At metaphase, the microsporocyte layer for the sterile anther has disappeared and resulted in full degradation as present in Figure $3 \mathrm{~F}$.

DNA FRAGMENTATION IN STERILE ANTHERS. The previously described phenotypic analysis by observation of TEM suggests that the mutation in sterile anthers affected the development of both microsporocyte and tapetal cells. To test whether the cell death of the anthers in the CMS line GP1BC1-12 was simultaneously accompanied the cleavage of nuclear DNA, we performed TUNEL assay for sterile anthers with fertile anthers as control. Three developmental stages of anthers were analyzed, including the microsporocyte stage, meiosis stage, and metaphase. During the microsporocyte stage, both the fertile anthers and the sterile anthers showed TUNEL-negative nuclei, indicating lack of DNA fragmentation at this stage (Fig. 3A and D). In the meiosis stage, all outer cell layers except the microsporocyte in the sterile anthers showed strong staining, suggesting that the nuclear DNA of the sterile anthers was cleaved (Fig. 3B and E). However, strong TUNEL-positive signals of fertile 


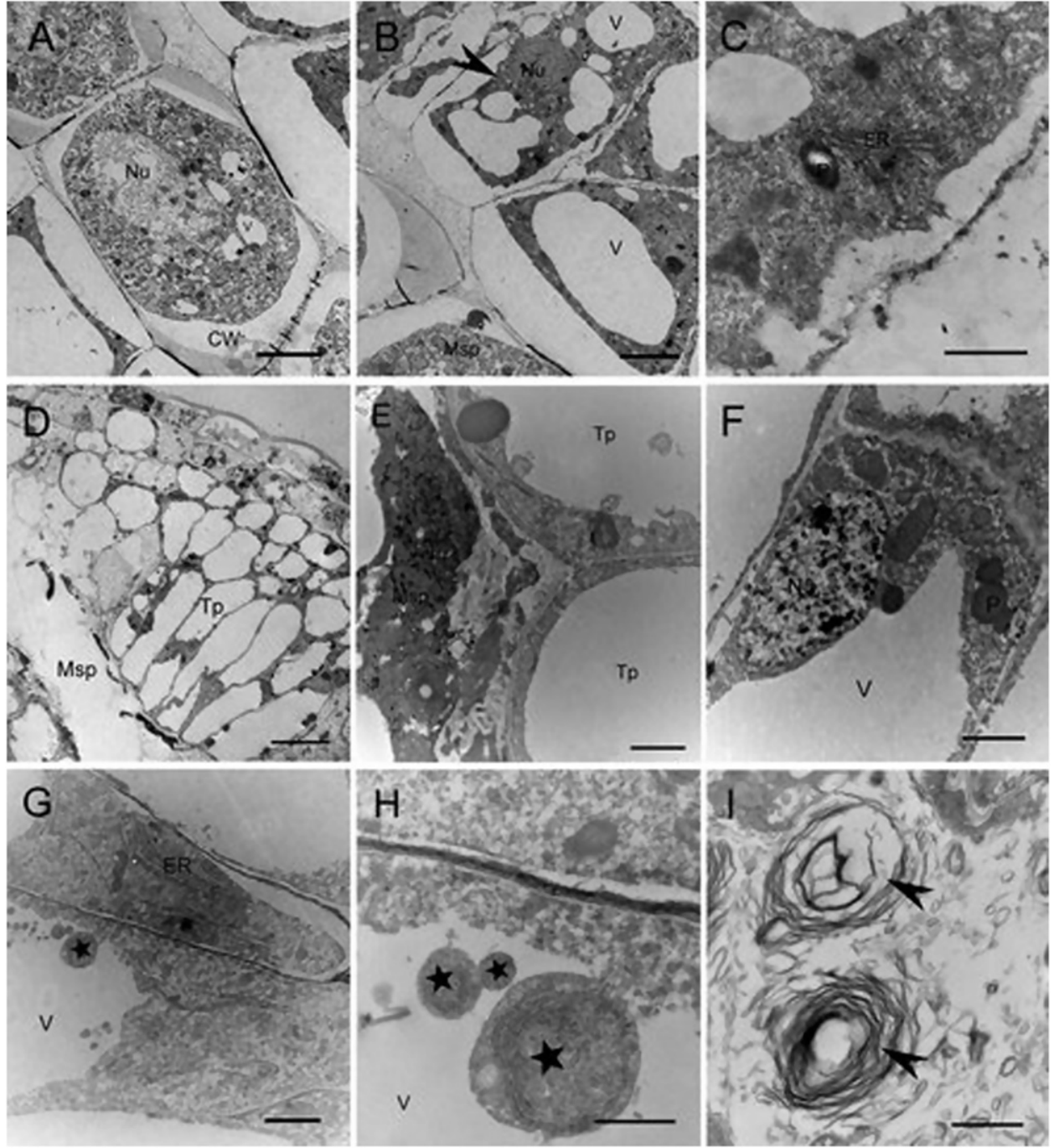

Fig. 2. Abnormal structure shown in microsporocyte and tapetal cell at the meiosis stage of Platycodon grandiflorum sterile anther. The structures were observed by transmission electron microscope at the meiosis stage of anthers from cytoplasmic male-sterile line GP1BC1-12 and maintainer fertile line GS902. In fertile anther, the microsporocyte had integral structure (A), and its tapetal cell with a little shrinkage (arrow) had a dense cytoplasm (B). However, in sterile anther, microsporocyte had been almost degenerated except for a little vestigium left. Some tapetal cells degenerated, and other tapetal cells with a comparatively integral structure had an extremely expanded vacuolar (D). No structure of any organelles could be discerned in its microsporocyte vestigium (E), and chromatin aggregation was present in these tapetal cells with integral structure in sterile anther $(\mathbf{F})$. The endoplasmic reticulum (ER) of tapetal cell was short in fertile anther $(\mathbf{C})$ but was clustered and elongated in sterile anther $(\mathbf{G})$. Vesicles (asterisk) arose from the edge of vacuole $(\mathbf{G})$, and some matter like ER (asterisks) was present in them $(\mathbf{H})$. Two multilamellar vesicles (arrows) and debris of organelles were present in the tapetal cell during degradation (I). Scale bars are $5 \mu \mathrm{m}$ for $\mathbf{A}$ and $\mathbf{B} ; 2 \mu \mathrm{m}$ for $\mathbf{E}, \mathbf{F}$, and $\mathbf{G} ; 1 \mu \mathrm{m}$ for $\mathbf{C}$ and $\mathbf{I} ; 10 \mu \mathrm{m}$ for $\mathbf{D}$; and $0.5 \mu \mathrm{m}$ for $\mathbf{H} ; \mathbf{C W}=$ cell wall; $\mathbf{P}=$ plastid; Tp = tapetum; Msp = microsporocyte; $\mathrm{Nu}=$ nucleus; $\mathrm{V}=$ vacuole.

anthers did not appear in the tapetal and connective cells until in the metaphase for the sterile anthers (Fig. 3C and F). Therefore, it may be concluded that DNA cleavage in the sterile anthers occurred earlier than that in the fertile anthers.

\section{Discussion}

The abortion of anthers in the CMS line GP1BC1-12 AND AUTOPHAGY. Autophagy occurs by the uptake of cytoplasmic constituents into the vacuole, where they are degraded by vacuolar processing enzymes. Two ways are involved, macroautophagy and microautophagy (Bassham, 2007; Bassham et al.,
2006; Hara-Nishimura and Hastsugai, 2011). In our study, macroautophagy and microautophagy both occurred during anther abortion of the CMS line GP1BC1-12.

Macroautophagy is initiated in the cytoplasm with the formation of cup-shaped membranes of unknown source that enclose the material to be degraded. The outer membrane of the autophagosome fuses with the tonoplast, and the internal vesicle is released into the vacuole where the degradation of its membrane and internal remnants is completed (Bassham, 2007; Bassham et al., 2006). The macroautophagic pathway is induced in vacuolated cells to adapt to various environmental stresses such as nutrient starvation and water stresses (Bassham, 2007; Bassham et al., 2006; Cao and Li, 2010). In our study on sterility, the internal vesicles in which an ER-like structure is sequestered (Fig. 2H) were detected in the tapetal cell of the CMS line GP1BC1-12. Similar internal vesicle-like structures have been observed in the tapetal cells of the $A$. thaliana ms 1 sterile line in the microspore stage (Vizcay-Barrena and Wilson, 2006).

Except for internal vesicles of autophagosomes in the tapetal cell of the CMS line GP1BC1-12, the rearrangement of the ER in which part of the cytoplasm or organelles are sequestered is another feature in the sterile microsporocytes (Fig. 1E) and tapetal cells (Figs. 1H and 2G). The inclusion and concentric arrangement of the ER were often observed in root cells under stresses (Cao and Li, 2010; Davies et al., 1987) and also accompanied the course of gametophyte abortion, such as the tapetum of Phaseolus vulgaris under heat stress, the ovule of $A$. thaliana under water stress, the tapetum in the male-sterile anthers of Conslea, and microsporocytes in the male-sterile anthers of B. napus (Hauser et al., 2006; Strittmatter et al., 2006; Suzuki et al., 2001; Wu et al., 2009a). In fact, the rearrangement of the ER is considered to be related to the macroautophagic process. Studies indicated that ER rearrangement would activate the autophagic pathway, where autophagosomes in eukaryotes would be formed by surrounding organelles of the ER (Affenzeller et al., 2009; Cao and Li, 2010; Ogata et al., 2006).

In microautophagy, material is directly engulfed by the vacuole through invagination of the tonoplast followed by pinching off of the membrane to release a vesicle containing the cytoplasmic constituents inside the vacuole lumen. In our 

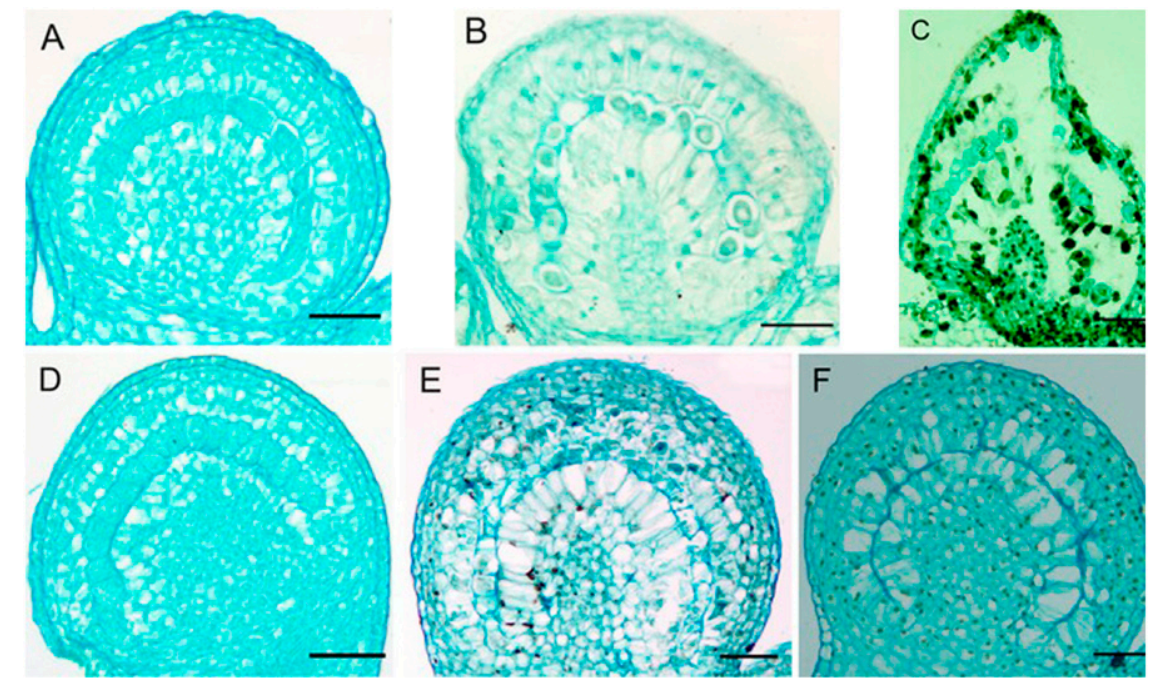

Fig. 3. DNA fragments in fertile and sterile anthers of Platycodon grandiflorum. The fixed and embedded anthers from cytoplasmic male-sterile line GP1BC1-12 and maintainer fertile line GS902 were assayed using a terminal deoxynucleotidyl transferase-mediated deoxyuridine triphosphate nick end labeling (TUNEL) kit and observed under a light microscope. No positive signal was present in the fertile anther at the microsporocyte stage (A) and the same was in the sterile anther (D). No significant positive signal was detected in the fertile anther at the meiosis stage (B) but was detected in the sterile anther at this stage (E). Positive signals were detected in both fertile anther wall $(\mathbf{C})$ and sterile anther wall $(\mathbf{F})$. Nuclei stained with fast green is indicated by green color, whereas nut brown to black is TUNEL-positive staining. Scale bar is $50 \mu \mathrm{m}$.

study, the cavity of vacuoles in the microsporocytes of sterile anthers and the mitochodria engulfed by the vacuole of tapetal cells (Fig. 1E and G) indicated that microautophagy occurred. Microautophagic-like processes have been observed during the deposition of storage proteins in developing seeds of Triticum aestivum and T. turgidum and release of rubber particles into the vacuole. The processes were also detected in some species during seed germination for degradation of starch granules and storage of protein in vacuoles (Bassham, 2007). However, the processes are seldom noted during sterility like in our study.

The autophagic vesicle, the abnormal arrangement of the $E R$, and the invagination of the vacuole provided evidence that autophagy was accompanied with anther abortion of the CMS line GP1BC1-12, which is similar to the PCD in plants that showed typical morphological features of autophagy (Bassham, 2007). Besides the three structural features mentioned, lamellar and multilamellar detected in the voculars of tapetal cells were seemingly related to the macroautophagy and they may be the intermediate product of autophagy. However, further evidence was needed to verify this speculation.

The abortion OF ANTHERS IN THE CMS LINE GP1BC1-12 AND APOPTOSIS. Apoptosis also occurred in the tapetum of the CMS line GP1BC1-12, which was manifested by the DNA cleavage in the tapetal cells (Fig. 3E) and chromatin aggregation (Figs. 1E and 2D) in both microsporocytes and tapetal cells. As the hallmark of apoptosis, DNA cleavage was frequently reported in tapetal cells of sterile anthers such as the B. napus line 9012A (Wan et al., 2010), PET1-CMS H. annuus (Balk and Leaver, 2001), PSGMS O. sativa 58S (Shi et al., 2009) and api5-1 O. sativa (Li et al., 2011), and also reported in microspores of the $A$. thaliana ms1 sterile line and the honglian CMS line of $O$. sativa (Li et al., 2003; Vizcay-Barrena and Wilson, 2006). Chromatin aggregation, present in both abortive microsporocytes and tapetal cells of GP1BC1-12, is another feature of apoptosis and was described in the meiocyte of CMS in

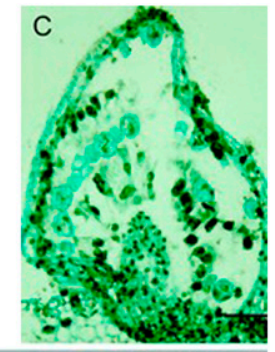

Brachiaria hybrid (Fuzinatto et al., 2007) and tapetal cells in sterile anthers (Love et al., 2008; Papini et al., 1999).

Different cell death paths are seldom noted simultaneously in one cell of a plant, but they have been observed in animals such as in the salivary gland of Drosophila melanogaster during developmental cell death and hela cells with loss of LAMP2 sterile (González-Polo et al., 2005; Lee and Baehrecke, 2001). Maybe, autophagy and apoptosis play different roles in decision cell death or survival. When related studies move forward, more cell death phenomena through different pathways may be observed.

PCD IN THE CMS LINE GP1BC1-12 AND ABNORMALITY OF MITOCHONDRIA. The result of TEM observation and the TUNEL assay showed that autophagy and apoptosis of microsporocytes and tapetal cells directly take part in the male sterility of the CMS line GP1BC1-12.

In fact, expression of novel open reading frames that originated by aberrant recombination events of the mitochondria genome is generally considered as the cause leading to CMS (Budar and Pelletier, 2001; Schnable and Wise, 1998).

The mitochondrial permeability transition (MPT) is the key factor to connect the cell death paths and the sterility of the mitochondrial gene. MPT is defined as an increase in the permeability of mitochondrial membranes, which involved with molecules of less than $1500 \mathrm{D}$ in molecular weight, and has been taken as a common mechanism of autophagy, apoptosis, and necrosis (Lemasters et al., 1998, 2009). Seemingly, the abnormal expression of the genome in mitochondria in the abortive anthers would result in CMS through MPT, which has been verified in the PET-CMS H. annuus and Honglian CMS O. sativa (Balk and Leaver, 2001; Li et al., 2003). In our study, the inner membrane of the mitochondria of the microsporocyte stage was severely damaged (Fig. 1H), and MPT, which would lead to PCD, must have been induced as that in Honglian CMS. This assumption would be verified further and the sterile genes of mitochondria leading to MPT in the CMS line GP1BC1-12 should be screened.

\section{Literature Cited}

Affenzeller, M.J., A. Darehshouri, A. Andosch, C. Lutz, and U. LutzMeindl. 2009. PCD and autophagy in the unicellular green alga Micrasterias dentuculata. Autophagy 5:853-855.

Aubert, S., E. Gout, R. Bligny, D. Marty-Mazars, F. Barrieu, J. Alabouvette, F. Marty, and R. Douce. 1996. Ultrastructural and biochemical characterization of autophagy in higher plant cells subjected to carbon deprivation: Control by the supply of mitochondria with respiratory substrates. J. Cell Biol. 133:1251-1263.

Balk, J. and C.J. Leaver. 2001. The PET1-CMS mitochondrial mutation in sunflower is associated with premature programmed cell death and cytochrome c release. Plant Cell 13:1803-1818.

Bassham, D.C. 2007. Plant autophagy-More than a starvation response. Curr. Opin. Plant Biol. 10:587-593. 
Bassham, D.C., M. Laporte, F. Marty, Y. Moriyasu, Y. Ohsumi, and L.J. Olsen. 2006. Autophagy in development and stress responses of plants. Autophagy 2:2-11.

Budar, F. and G. Pelletier. 2001. Male sterility in plants: Occurrence, determinism, significance and use. Life Sci. 323:533-550.

Cao, M.M. and X. Li. 2010. Die for living better-Plants modify root system architecture through inducing PCD in root meristem under severe water stress. Plant Signal. Behav. 5:1635-1636.

Choi, Y.H., D.S. Yoo, M.R. Cha, C.W. Choi, Y.S. Kim, S.U. Choi, K.R. Lee, and S.Y. Ryu. 2010. Antiproliferative effects of saponins from the roots of Platycodon grandiflorum on cultured human tumor cells. J. Nat. Prod. 73:1863-1867.

Coimbra, S., L. Torrão, and I. Abreu. 2004. Programmed cell death induces male sterility in Actinidia deliciosa female flowers. Plant Physiol. Biochem. 32:537-541.

Davies, D.D., P. Kenworthy, B. Mocquot, and K. Roberts. 1987. The effects of anoxia on the ultrastructure of pea roots, p. 265-277. In: Crawford, R.M.M. (ed.). Plant life in aquatic and amphibious habitats. Blackwell Press, Oxford, UK.

Edinger, A.L. and C.B. Thompson. 2004. Death by design: Apoptosis, necrosis and autophagy. Curr. Opin. Cell Biol. 16:663-669.

Fuzinatto, V.A., M.S. Pagliarini, and C.B. Valle. 2007. Evidence of programmed cell death during microsporogenesis in an interspecific Brachiaria (Poaceae: Panicoideae: Paniceae) hybrid. Genet. Mol. Res. 6:308-315.

González-Polo, R.A., P. Boya, A.L. Pauleau, A. Jali, N. Larochett, S. Souquère, E.L. Eskelinen, G. Pierron, P. Saftig, and G. Kroemer. 2005. The apoptosis/autophagy paradox: Autophagic vacuolization before apoptotic death. J. Cell Sci. 118:3091-3102.

Greenberg, J.T. 1996. Programmed cell death: A way of life for plants. Proc. Natl. Acad. Sci. USA 93:12093-12097.

Greenberg, J.T. 1997. Programmed cell death in plant pathogen interactions. Annu. Rev. Plant Physiol. Plant Mol. Biol. 38:525535.

Halevy, A.H., E. Shlomo, and O. Ziv. 2002. Improving cut flower production of balloon flower. HortScience 37:759-761.

Hara-Nishimura, I. and N. Hastsugai. 2011. The role of vacuole in plant cell death. Cell Death Differ. 18:1298-1303.

Hauser, B.A., K.L. Sun, D.G. Oppenheimer, and T.L. Sage. 2006. Changes in mitochondrial membrane potential and accumulation of reactive oxygen species precede ultrastructural changes during ovule abortion. Planta 223:392-399.

Hayward, A.P., J.T. Sao, and S.P. Dinesh-Kumar. 2009. Autophagy and plant innate immunity: Defense through degradation. Semin. Cell Dev. Biol. 20:1031-1037.

Henming, W. and Y.C. Alice. 2000. Programmed cell death in plant reproduction. Plant Mol. Biol. 33:267-281.

Kang, Y.S., K.P. Hong, D.C. Jung, S. Hong, J.H. Lee, S.Y. Nah, Y. Lim, and D.H. Bae. 2008. Calcium channel-blocking activity of chinese balloon flower (Platycodon grandiflorum) functional foods. Food Sci. Biotechnol. 17:156-160.

Krishnamurthy, K.V., R. Krishnaraj, R. Chozhavendan, and F.S. Christopher. 2000. The programme of cell death in plants and animals-A comparison. Curr. Sci. 79:1169-1181.

Ku, S., H. Yoon, H.S. Suh, and Y.Y. Chung. 2003. Male-sterility of thermosensitive genic male-sterile rice is associated with premature programmed cell death of the tapetum. Planta 217:559-565.

Lee, C.Y. and E.H. Baehrecke. 2001. Steroid regulation of autophagic programmed cell death during development. Development 128: 1333-1355.

Lemasters, J.J., A.L. Nieminen, T. Qian, L.C. Trost, S.P. Elmore, Y. Nishimura, R.A. Crowe, W.E. Cascio, C.A. Bradham, D.A. Brenner, and B. Herman. 1998. The mitochondrial permeability transition in cell death: A common mechanism in necrosis, apoptosis and autophagy. Biochim. Biophys. Acta 1366:177-196.

Lemasters, J.J., T.P. Theruvath, Z. Zhong, and A.L. Nieminen. 2009. Mitochondrial calcium and the permeability transition in cell death. Biochim. Biophys. Acta 1787:1395-1401.
Li, N., D.S. Zhang, H.S. Liu, C.S. Yin, X.X. Li, W.Q. Liang, Z. Yuan, B. Xu, H.W. Chu, J. Wang, T.Q. Wen, W. Huang, D. Luo, H. Ma, and D.B. Zhang. 2006. The rice tapetum degeneration retardation gene is required for tapetum degradation and anther development. Plant Cell 18:2999-3013.

Li, S.Q., C.X. Wan, J. Kong, Z.J. Zhang, Y.S. Li, and Y.G. Zhu. 2003. Programmed cell death during microgenesis in honglian CMS line of rice is correlated with oxidative stress in mitochondria. Funct. Plant Biol. 31:369-376.

Li, X., X. Gao, Y. Wei, L. Deng, Y. Ouyang, G. Chen, X. Li, Q. Zhang, and C. Wu. 2011. Rice APOPTOSIS INHIBITOR5 coupled with two DEAD-box adenosine 5'-triphosphate-dependent RNA helicases regulates tapetum degeneration. Plant Cell Online 23:1416-1434.

Liu, D.J. and W.X. Ma. 2001. Platycodon grandiflorum. Chinese Traditional Medicine Publ. House, Beijing, China.

Love, A.J., J.J. Milner, and A. Sadanandom. 2008. Timing is everything: Regulatory overlap in plant cell death. Trends Plant Sci. 13:589-595.

Ogata, M., S.I. Hino, A. Saito, K. Morikawa, S. Kondo, S. Kanemoto, T. Murakami, M. Taniguchi, I. Tanii, K. Yoshinaga, S. Shiosaka, J.A. Hammarback, F. Urano, and K. Imaizumi. 2006. Autophagy is activated for cell survival after endoplasmic reticulum stress. Mol. Cell. Biol. 26:9220-9231.

Papini, A., S. Mosti, and L. Brighigna. 1999. Programmed-cell-death events during tapetum development of angiosperms. Protoplasma 207:213-221.

Park, B., N. Oliveira, and S. Pearson. 1998. Temperature affects growth and flowering of the balloon flower [Platycodon grandiflorum (Jacq.) A. DC. Astra Blue]. HortScience 33:233-236.

Patel, S.J., R.T. Desai, R.S. Bhakta, D.U. Patel, V.C. Kodappully, and S.C. Mali. 2009. Heterosis studies in cowpea [Vigna unguiculata (L.) Walp]. Legume Res. 32:199-202.

Pennell, R. and C. Lamb. 1997. Programmed cell death in plants. Plant Cell 9:1157-1168.

Pradhan, A.K., Y.S. Sodhi, A. Mukhopadhyay, and D. Pental. 1993. Heterosis breeding in India mustard (Brassica juncea L. C zern \& Coss): Analysis of component characters contributing to heterosis for yield. Euphytica 69:219-229.

Reape, T.J., E.M. Molony, and P.F. McCabe. 2008. Programmed cell death in plants: Distinguishing between different modes. J. Expt. Bot. 59:435-444.

Schnable, P. and R.P. Wise. 1998. The molecular basis of cytoplasmic male sterility and fertility restoration. Trends Plant Sci. 3:175-180.

Shi, F.H., C. Sui, C.M. Yang, J.H. Wei, Y. Jin, H.J. Chen, L.Q. Huang, Y.K. Zhao, X.S. Zhao, Q.L. Chu, and J. Yu. 2011. Development of a stable male-sterile line and its utilization in high yield hybrid of Platycodon Grandiflorum. J. Medicinal Plants Res. 5:3388-3399.

Shi, Y.L., S. Zhao, and J.L. Yao. 2009. Premature tapetum degeneration: A major cause of abortive pollen development in photoperiod sensitive genic male sterility in rice. J. Integr. Plant Biol. 51:773781.

Song, C.Y., M.S. Roh, S.K. Chung, and R.H. Lawson. 1993. Effect of temperature and light on growth and flowering of potted plant production of platycodon. J. Korea Soc. Hort. Sci. 33:336-353.

Strittmatter, L.I., V. Negron-Ortiz, and J. Hickey. 2006. Comparative microsporangium development in male-fertile and male-sterile flowers of Consolea (Cactaceae): When and how does pollen abortion occur. Grana 35:81-100.

Suzuki, K., H. Takeda, T. Tsukaguchi, and Y. Egawa. 2001. Ultrastructural study on degeneration of tapetum in anther of snap bean (Phaseolus vulgaris L.) under heat stress. Sex. Plant Reprod. 13:293299.

Van-Doorn, W.G., E.P. Beers, J.L. Dangl, V.E. Franklin-Tong, P. Gallois, I. Hara-Nishimura, A.M. Jones, M. Kawai-Yamada, E. Lam, J. Mundy, L.A. Mur, M. Petersen, A. Smertenko, M. Taliansky, F. Van Breusegem, T. Wolpert, E. Woltering, B. Zhivotovsky, and P.V. Bozhkov. 2011. Morphological classification of plant cell deaths. Cell Death Differ. 18:1241-1246. 
Van-Doorn, W.G. and E.J. Woltering. 2005. Many ways to exit? Cell death categories in plants. Trends Plant Sci. 10:1360-1385.

Vaux, D.L. and S.J. Korsmeyer. 1999. Cell death in development. Cell 96:235-253.

Vizcay-Barrena, G. and Z.A. Wilson. 2006. Altered tapetal PCD and pollen wall development in the Arabidopsis ms1 sterile. J. Expt. Bot. 57:2709-2717.

Vogel, K.P. and R.B. Mitchell. 2008. Heterosis in switchgrass: Biomass yield in swards. Crop Sci. 38:2159-2163.

Wan, L.L., X.Y. Xia, D.F. Hong, J. Li, and G.S. Yang. 2010. Abnormal vacuolization of the tapetum during the tetrad stage is associated with male sterility in the recessive genic male sterile Brassica napus L. Line 9012A. J. Plant Biol. 53:121-133.

Wu, H.J., X.H. Liu, K. Chen, Z.P. Cai, X.J. Luo, and T. Zhang. 2009a. Disintegration of microsporocytes in a male sterile sterile of Brassica napus L. is possibly associated with endoplasmic reticulum-dependent autophagic programmed cell death. Euphytica 170:263-273.

Wu, H.M. and A.Y. Cheung. 2000. Programmed cell death in plant reproduction. Plant Mol. Biol. 33:267-281.

Wu, J.R., Y.Z. Yan, X.L. Quan, J. Piao, and S.Q. Wu. 2008. JXB-1 discovery and identification of male sterility Platycodon grandiflorum germplasm. Bull. Bot. Res. 28:716-719.

Wu, S.Q., X.L. Quan, X.M. Piao, Y.Z. Yan, and J.R. Wu. 2009 b. Morphological study on anther development of male sterile of platycodon grandiflorum. Guihaia 29:729-731.

Wyllie, A.H. 1980. Glucocorticoid-induced thymocyte apoptosis is associated with endogenous endonuclease activation. Nature 283: $555-556$

Zhang, J., Q.H. Dong, K. Yang, Q.Q. Cao, Y.Q. Feng, and L. Qin. 2007. Studies on the ultrastructure of abnormal cell death during sterile short catkin development in chestnut. Acta Hort. Sinica 33:605-608. 